\title{
THE DIGITALIZATION OF STEEL PRODUCTION *
}

\author{
KURT HERZOG ${ }^{1}$ \\ GÜNTHER WINTER ${ }^{2}$ \\ GERHARD KURKA ${ }^{1}$ \\ KAI ANKERMANN ${ }^{2}$ \\ RAFFAEL BINDER ${ }^{3}$ \\ MARKUS RINGHOFER ${ }^{1}$ \\ ANDREAS MAIERHOFER ${ }^{2}$
}

\begin{abstract}
State-of-the-art automation information technology and connectivity enables the digitalization of steel production that goes far beyond conventional automation of industrial production. Initiatives have been started around the globe to foster digitalization like IloT (Industrial Internet of Things) in the US, Industrie 4.0 in Germany or China 2025. Primetals Technologies is actively driving the digitalization shaping the future of steel production.

Intelligent combination of sensor technology combined with digital models as well as quality and production planning and control systems leads to new dimensions in product quality and production cost reduction.

New diagnostic ways allow intuitive fault tracing or processing to support faster maintenance. Digital assistants support both operation and maintenance using context-oriented or self-learned information.
\end{abstract}

Keywords: Digitalization, Integration, Productivity, Digital Assistant.

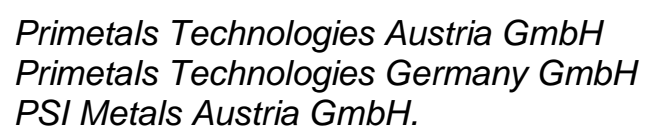




\section{INTRODUCTION}

The technological developments of the recent years in the areas of communication technologies and IT, both hardware and software, allow handling a complexity at formerly impossible extent. The production process of steel is a very complex process, and therefore, the application of new technologies in the field of steel production is already supporting and can further support to optimize the entire production chain including the integration of business processes along the life-cycle of production facilities. Initiatives supporting the digitalization of industries have been started around the globe. Industrial loT [1] in the US, Industrie 4.0 [2] in Germany or Chinese Made in China 2025 [3] are some well-known initiatives reflecting the industrial transformation. Although these concepts are slightly different, they have one thing in common: The concepts are generic and need to be specified and applied in a more specific way in the different industries.

In the following, a way how these concepts can be applied in the steel industry is drafted and possible benefits are shown.

\begin{tabular}{|c|c|c|}
\hline $\begin{array}{l}\text { STEEL PRODUCERS } \\
\text { REQUIREMENTS }\end{array}$ & & NEW TECHNOLOGIES \\
\hline End-customer integration & QUALITY & Communication technologies \\
\hline Lot-size 1 & & Computational power \\
\hline New business models & & Internet technologies \\
\hline Know-how management & & Cloud technologies \\
\hline Supplier integration & & Big data analytics \\
\hline New steel grades & & Self-learning systems \\
\hline Availability of information & & Mobile devices \\
\hline Ergonomy/operator support & FLEXIBILITY PRODUCTIVITY & Augmented reality \\
\hline
\end{tabular}

\section{ACHIEVED BY HOLISTIC AUTOMATION}

Figure 1: New technologies enable the fulfilling of many steel producer's requirements

The central question is, what is the steel industry expecting from the digitalization?

One example may go into the direction of just-in-time production. Selling of the final product will take place via Internet, may be even via Trading-Platforms not operated by the steel producer. To be competitive in such an environment, it is necessary that small batch sizes can be produced with minimal lead time and further a wide range of steel-grades and products can be supplied. The pressure on product prices and production cost will stay as high as it is or will even increase. This requires, that all possibilities to reduce production costs, productivity and product quality have to be 
exploited. The new technologies can contribute a lot in this area. So the target is, to use all available technologies to fulfil the requirements of the steel producers to survive and be successful on the long run.

As shown in Figure 1, the digitalization of steel production can be seen as the consequent application of new technologies to fulfil steel producers' requirements. The focus topics in all cases are Quality, Flexibility and Productivity.

Quality is conformance with requirement, allowing steel producers to achieve product quality with narrow tolerance bands and a high degree of reproducibility. It is expected that the importance of seamless documentation of the produced quality in each production step will increase further.

Flexibility means flexibility of production facilities and routes to produce a wide range of different steel grades and products in very small batch sizes. Flexibility can also mean flexibility in used raw materials. Depending on the market situation the production processes can be flexibly optimized towards different target values. Flexible adoption of the tap-to-tap time of a converter to the actual production needs can for example reduce the wear of the converter lining and therefore reduce production costs.

Productivity is reflected through its KPl's like throughput, yield, plant availability, operation and maintenance costs as some examples.

\section{The overall structure of the digitalized steel production - The Metals Orchestra}

To achieve highest quality, flexibility and productivity, all production units within the entire production chain (and beyond) need to be in harmony. Like in an orchestra, where all musicians need to perfectly play by themselves and with each other, also in the metals industry the individual production units need to be optimized but also need to interact to reach stated criteria. The concept of the Metals Orchestra is reflecting the requirements for a fully digitalized facility in the metals industry.

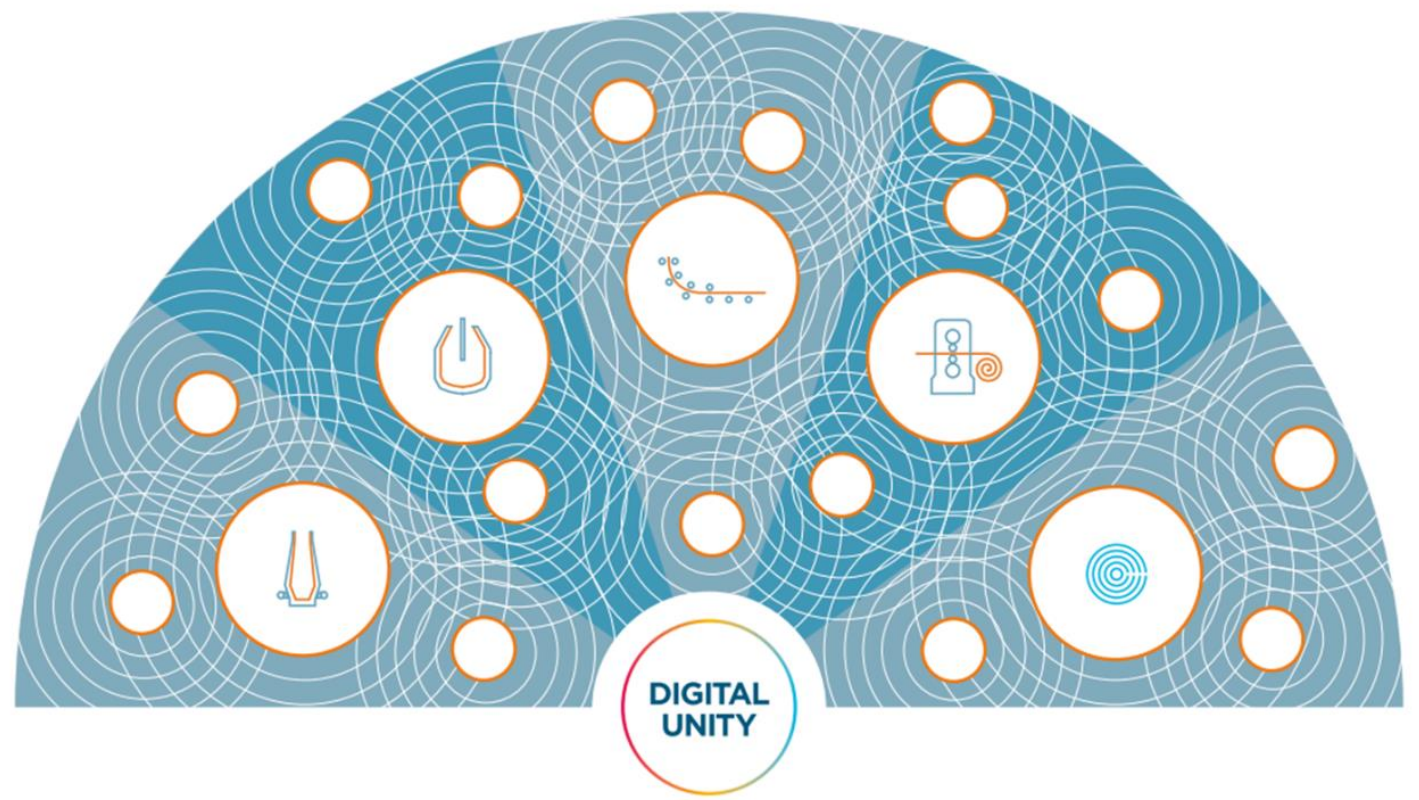

Figure 2: The Metals Orchestra 
In an excellent orchestra each individual musician needs to be perfectly trained, needs a good and perfectly maintained instrument and he needs the right music sheet. In the steel production, each individual production facility needs to fulfil certain requirements to participate in a digitalized production as shown in Figure 2.

Today steel production is already automated to a certain extent, however, often the systems in place work in an isolated way or with limited interfaces to other systems. The major challenge in digitalization is the integration of all systems and productions units. This integration takes place in three different dimensions and will be further described in the following paragraphs:

- Vertical Integration - Integration of systems across the classic automation levels from the sensor to the ERP system

- Horizontal Integration - Integration of systems along the entire production chain

- Life-cycle integration - Integration along the entire life-cycle of a plant from basic engineering to decommissioning

\subsection{Fully automated and digitalized production units - Vertical integration}

The goals of vertical integration are:

- Perfect mastery of the local production process

- Networking of data from the sensor to the planning level as the basis for data analysis, model optimization and, in future, use of artificial intelligence

- Adaptive systems that optimize themselves to the current plant status

- Flexible response to disturbances or planning changes

- Robot and assistance systems to relieve control personnel by increasing the degree of automation

- Automated diagnostics

- Modern intuitive HMI for efficient operation and decision-making support (in the case of quality management and maintenance management or by online documentation, for example)

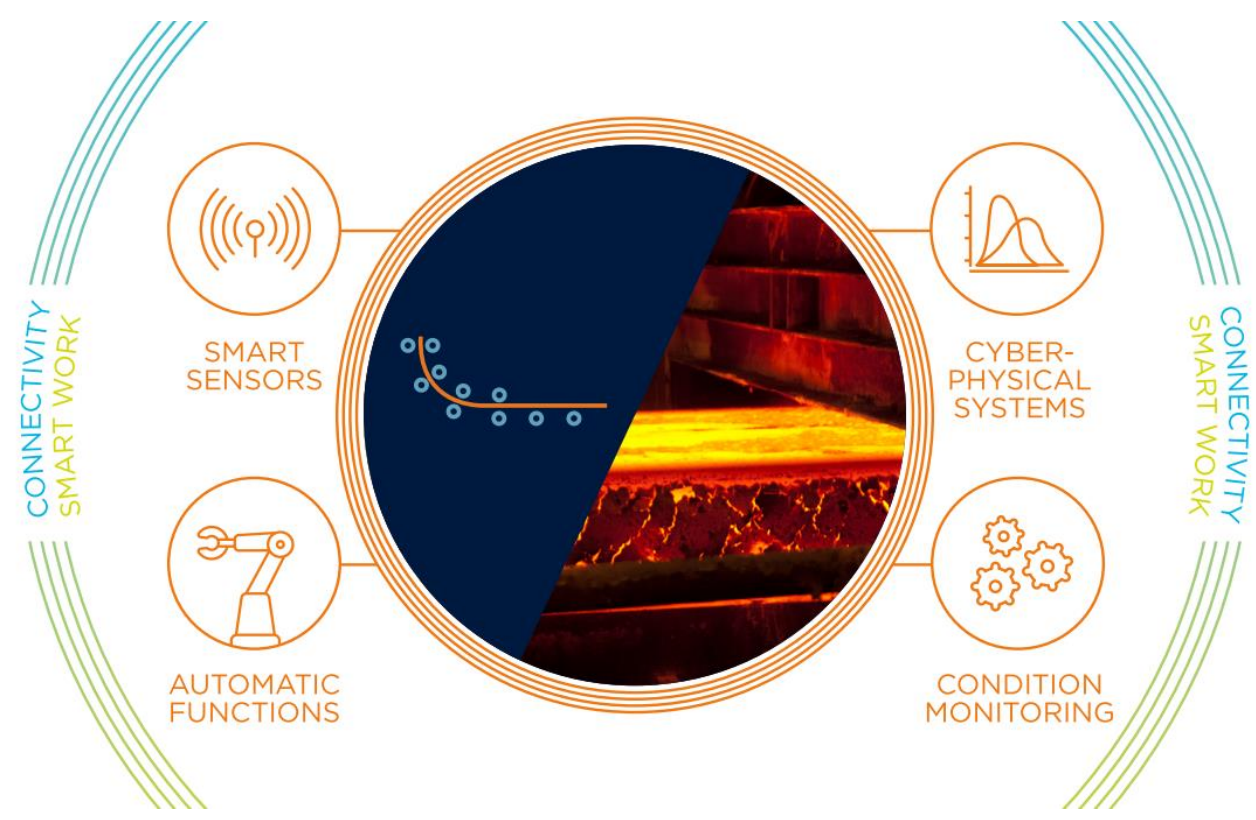

Figure 3: Core elements of a digitalized, automated plant 
The core elements of the digitalized plant as shown in Figure 3 are:

(Smart) Sensors: Sensors provide essential information about the process or the entire plant. They either directly measure physical values or use existing measurements to indirectly calculate additional information. This information is the enabler for the implementation of advanced automatic functions, process models as well as condition monitoring.

Automatic functions: Repetitive, labour - intensive and dangerous work is supported by fully-automated mechatronics solutions, robots and assistant systems.

Cyber-physical systems: So-called cyber-physical systems are the tight integration of the real world with a digital model. Figure 3 shows the example of a cyber-physical system for optimizing the material properties of hot strip in a cooling section.

It was specially developed for modern cooling sections with Power Cooling and high volumes of cooling water. The cyber-physical system is an ideal tool for the full

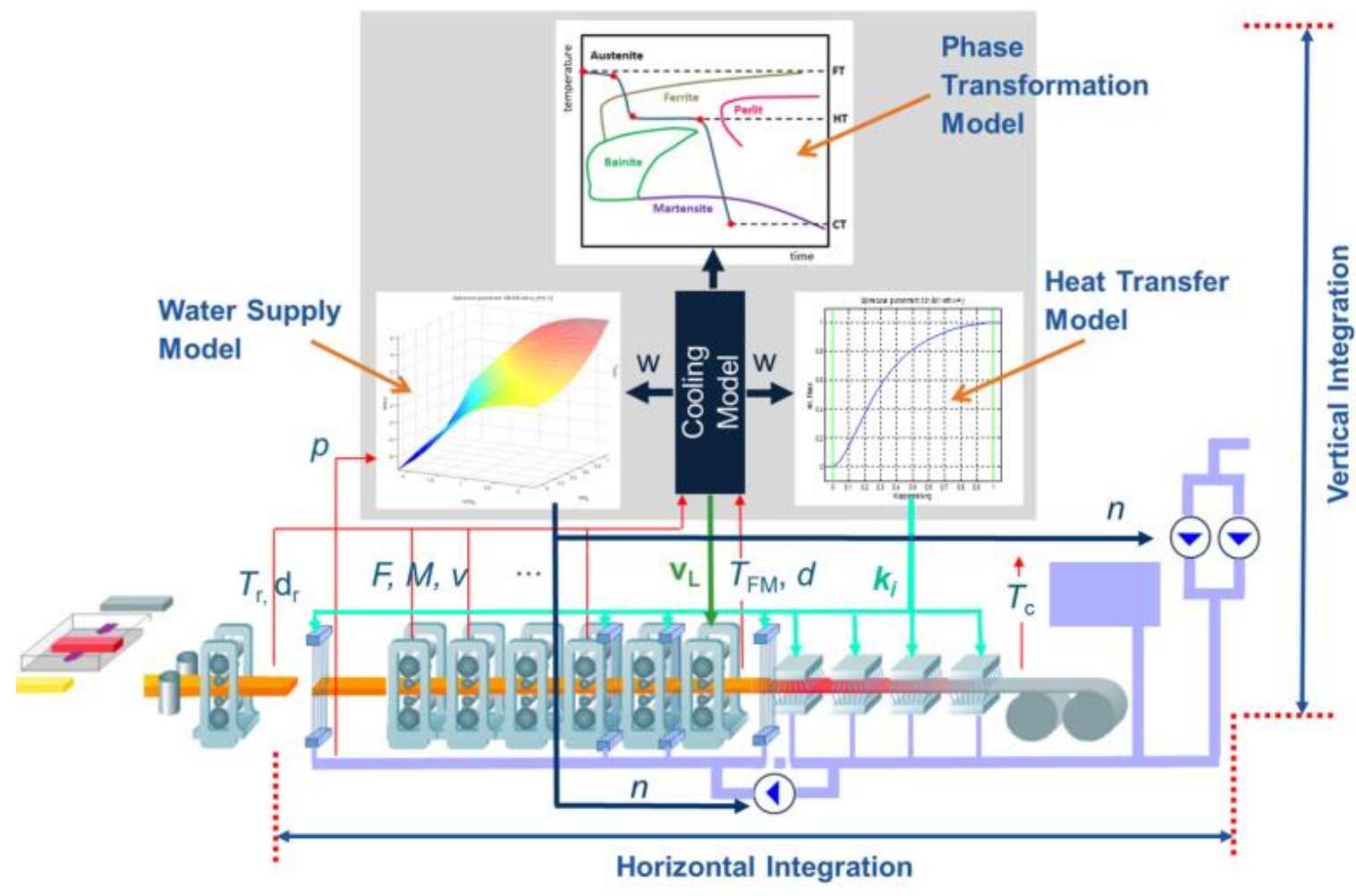

Figure 4: Cyber-Physical System Cooling Section

automatic flexible and low-cost production of a large variety of modern steel grades and dimensions on the hot strip mill:

- Flexible, expandable cooling strategy for pipe grades, dual-phase steels and high-carbon steels, for example

- Optimum technological coordination with the finishing mill

- Model-based holistic control of the entire cooling system through vertical integration 
- Highly dynamic plant operation with short response times for fast product changeovers

- Stable plant operation with high water volumes and strip thicknesses up to $25.4 \mathrm{~mm}$

- Stable plant operation in the low-temperature range

- Integrated microstructure monitor for monitoring material properties

Condition Monitoring: Comprehensive information about the condition of the equipment and the respective processes enables predictive maintenance, which will help to avoid unplanned outages. Enriched with data-based or mathematical models condition monitoring will develop to condition prediction in future.

Smart work: To ensure best possible operation and maintenance of a plant, a vast variety of information sources is required.

The core concept of "smart work" is, that all personnel will automatically receive exactly the information they need at the time to get their job done. Providing and maintaining this information along the entire life-cycle of a plant can be seen as the third dimension in integration: life-cycle integration.

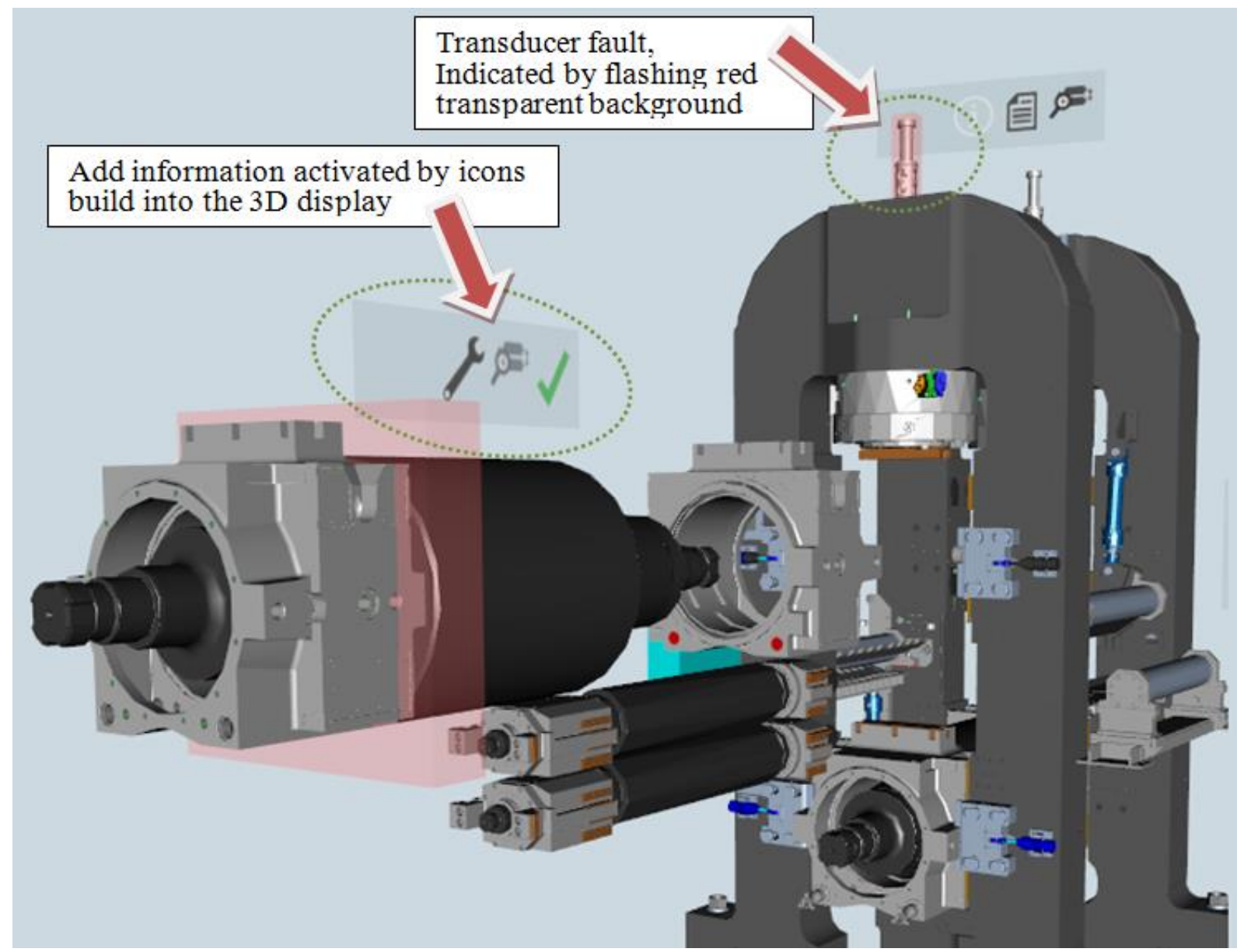

Figure 5: Example of a 3D Digital Maintenance Assistant 
One further aspect that needs to be considered in this context is the fact, that on the one hand increasing process-know-how is needed to produce high quality products, on the other hand the experience of producers vanishes with retiring or leaving of experienced operators and specialists.

Securing this know-how and providing it to operators and maintenance staff is essential.

Today's diagnostic systems usually provide only information about the current state of process or equipment. In the near future digital assistant systems will guide the operator or maintenance staff through their tasks with intuitive tools using digitalized knowledge and self-learning algorithms.

One example could be, that in case of a sensor fault 3D information about the location of a sensor, in addition with online documentation or instructions and direct links to the software are provided. 3D animations that show how to change the sensor, together with additional work instructions guide the maintenance staff through the repair process (Figure 5). Access to the documentation of former repairs ensure, that formerly collected know-how can be utilized.

The digital assistant will supply the operators with all information from material and process to electrical and mechanical equipment out of one system. For example they will receive an early warning about an upcoming problem together with all information how to overcome or react on the situation. This early warning will be generated by either mathematical models or self-learning methods. Service personnel will receive in parallel all information in which area this situation will occur and get automatically preselected documents up to information about availability of spare part on the stockyard. The comprehensive information will be presented on traditional HMl systems as well as on augmented reality devices.

New methods digitizing knowledge, developed and used already in other industries, will be introduced in the metals business. Natural Language Processing, Recurrent Neural Networks, are examples of new methods which will influence the operation in a complete new way in the future.

Connectivity: Advanced communication technologies allow for information to be transported beyond conventional limitations. From the collection of sensor data in harsh environments or from mobile equipment to displaying the information on mobile devices. From the shop floor to the "manager's pocket“.

\subsection{The Digital Unity - Horizontal Integration}

Each great orchestra needs an outstanding conductor. The conductor in the Metals Orchestra is the "Digital Unity" which is organizing the horizontal integration along the entire production chain. 


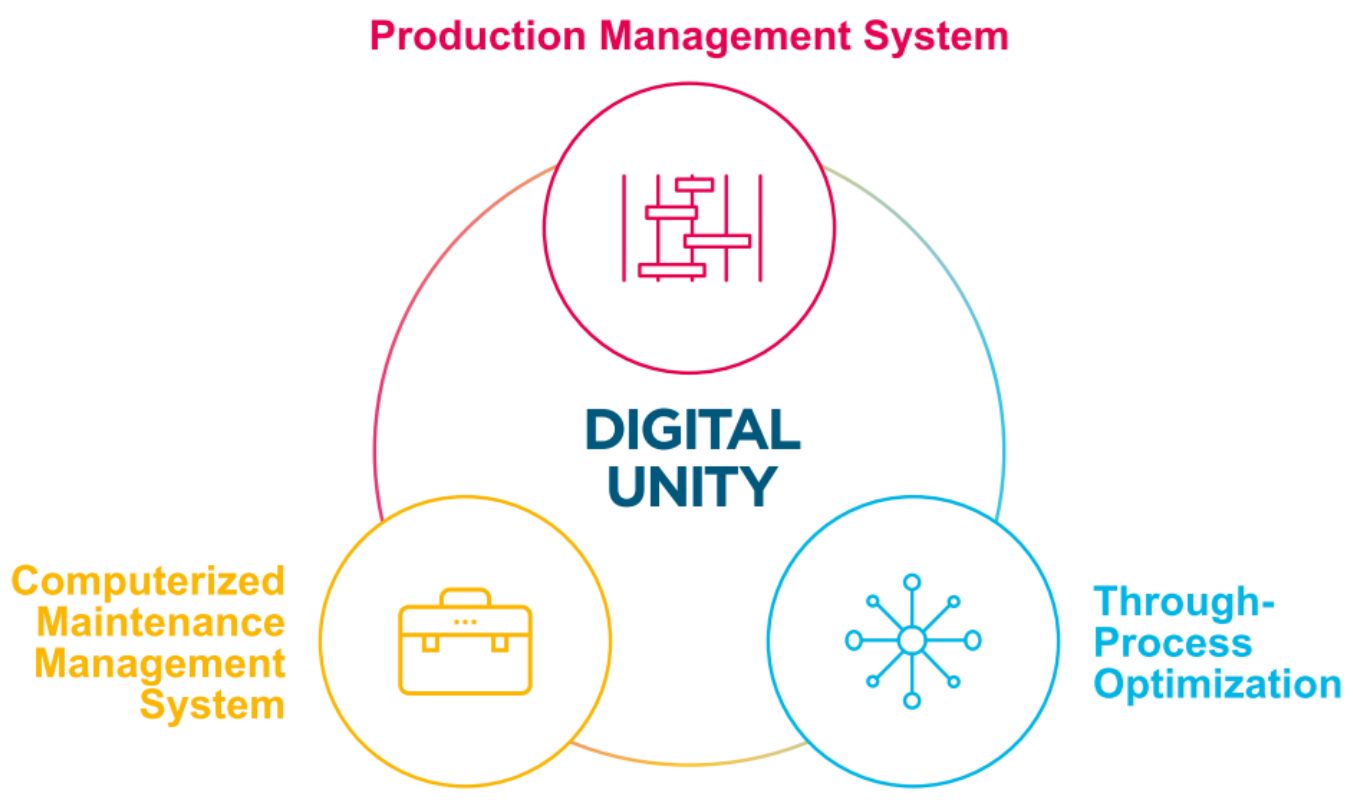

Figure 6: The Digital Unity

The objectives of the horizontal integration are:

- Perfect control and optimization of the entire process chain ("throughprocess")

- Expert systems for management of through-process know-how

- Integration throughout the entire value chain, also cross locations and industry wide

- Modern intuitive HMI to support humans in decision-making within the production process (planning, planning changes or reallocation)

The Digital Unity consists of the elements Production Management System, Through-Process Optimization and Computerized Maintenance Management System as shown in Figure 6.

Those systems are -partially - available and in operation. The novel aspect is, that these systems will strongly interact with each other as a unity, to further optimize production in a holistic manner.

\section{Production Management System}

The production management system covers the metals production process from iron and steel making, rolling till shipping of the final product. The Primetals Technology PMS production management solution is based on the product PSImetals, which provides a comprehensive set of modules specifically developed for the metals industry already proven in many installations.

Following the process, at first, it has to be evaluated, if and how a product can be produced with the available plant equipment. Therefore the "Product Design" requires extensive through process know-how and is in turn again the basis for Through Process Optimization during the actual production:

- Rules need to be defined that allow a dynamic translation of customer orders to production instructions including their processing route through 
the different production facilities and routes until shipment of the ordered product.

- Along the process intermediate products will be produced, which are also part of the design

- Technological target data for intermediate products are defined

- Definition of quality targets and quality envelopes

- Testing and sampling instructions to meet standards and customer requirements

Product specific information is combined with machine data and constraints and logistics information to compile all production relevant master data into a central repository.

This approach allows to dynamically follow a strategy of increased individualized customer products and allows ad-hoc statements on the feasibility to produce a required product.

The planning system has the ability to produce an order according the current utilization taking into account machine capacities and throughputs, planned maintenance and/or reduced machine capabilities.

Both aspects - the general feasibility to produce and the available capacity to fulfil required customer due dates - need to be available in real-time and de-central, to allow the sales department to act immediately.

\section{Through-Process Optimization}

Through Process Optimization (TPO) is an integrated know-how based concept developed by Primetals Technologies to improve efficiency and quality across all plants of a steel producer. The aim of TPO is to shorten time to market in case of cutting edge products. The digital platform of TPO is the Through-Process Quality Control (TPQC) system, which stores the expert know-how in terms of quality rules that are applied to all the gathered high resolution process data recorded across the entire production chain.

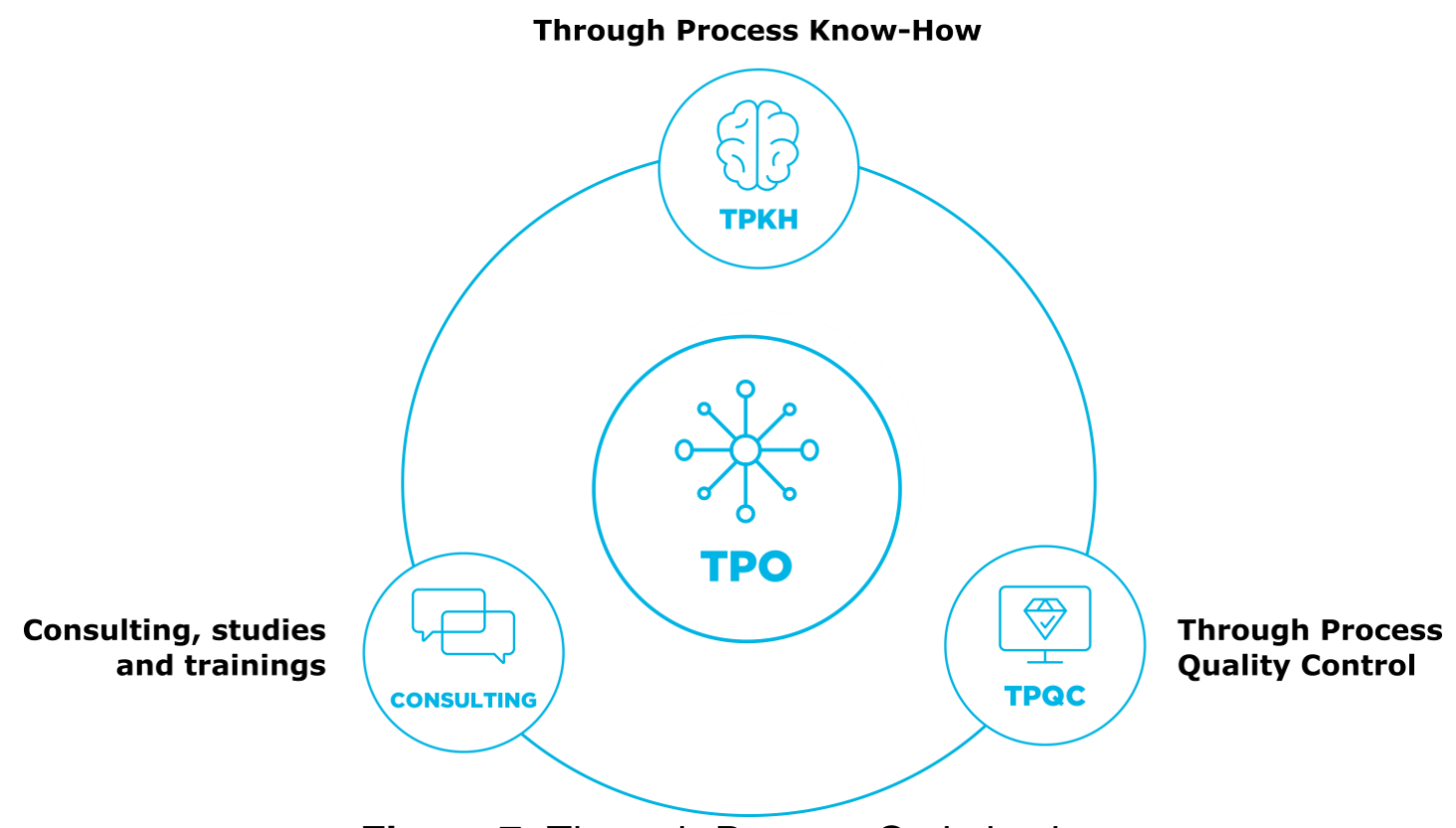

Figure 7: Through-Process Optimization 
The main parts of TPO comprise Through-Process Know How (TPKH) and ThroughProcess Quality Control (TPQC).

The main contribution of TPKH can be summarized as follows:

- Excellence in process and metallurgical assistance

- Product development of AHSS steel grades tailored to the plant capability

- Achieving and stabilizing best surface for exposed applications

- Implementation of rule based quality judgements and root cause analysis

- Specific expertise for process optimization supported by a pool of internal and external experts

- Assistance for production planning and trial runs and continuous process optimization

The TPQC system acts as a centralized data processing platform, which is gathering process and quality data from all plants covered by TPO consisting of the following main features:

- Product Explorer: in depth analysis of process data based on time or length of product

- Quality and process assistance: rule based quality checkpoints, root cause analysis and suggestions of corrective actions. Automatic rule based surface grading

- Surface defect density map of coils by the integration of all surface inspection systems

- Reporting: tailor made reporting

- Statistical Process Control (SPC) and Key Performance Indicators (KPIs): SPC to evaluate variations in the process and measure the process capability. KPIs for performance tracking for individual process units and the overall plant

- Business Intelligence functions: TPQC stores its KPIs and similar data for reporting in so called data cubes, which provide maximum flexibility for spread sheet evaluation and back office reporting.

\section{Computerized Maintenance Management System}

A Condition Monitoring System (CMS) is acquiring condition information in real-time from core components by means of traditional sensors or by the use of smart sensors. In addition condition information can be retrieved offline with mobile device (e.g. vibration measurements) and be feed into the CMS. As a result the current state of a machine is computed by using the retrieved information.

Planned production, driving upcoming machine utilization and type of products planned for production are considered when predicting machine's future condition. The machine's current condition and predicted future condition will be made available to the plant's Computerized Maintenance Management System (CMMS) as basis for the optimized, holistic planning of the maintenance actions and maintenance shutdowns. The maintenance plan and the production plan are harmonized between the CMMS and the PMS dynamically in order to realize the overall benefits described in the section aims above.

A recognized or predicted degradation in machine capability shall be considered by the production planning system to extend uptime e. $\mathrm{g}$. by rescheduling to reduce the load. 
The entire system shall be capable to react dynamically on sudden disturbances (i.e. deviations from normal plan or normal plant status) in an optimum way and assist the human operator in decision making.

The system can be installed in a Steel Plant as a whole or step-by-step as individuals parts.

\section{Implementation}

\section{Step-by-step to a digital plant}

The implementation of the digitalization is a process, that - in the steel industry started already years ago and it will be an ongoing process throughout the next years. There will not be one "off-the-shelf" solution covering all aspects of digitalization, but a stepwise implementation utilizing modules that may already be available on the market or will be developed in future. For the implementation, the focus has to be on the interoperability and connectivity of these modules to fit into an overall digitalization strategy. This digitalization strategy has to be derived from the business strategy by each steel producer individually, to support the business strategy to a maximum extent.

Plant builders and system suppliers are requested to develop and supply modular systems that support stepwise implementation of the steel producers' digitalization strategy and generate immediate benefit with each single module.

\section{Customer's benefit from implementing the Primetals Technologies Digital Unity}

The aforementioned close interaction between the players of the Digital Unity allow that any deviation of machine capability (e.g. throughput) and product constraints from standard machine definitions which are made available by the CMMS allow planning to react to those constraints immediately.

Planned downtimes are "negotiated" between production planning and CMMS automatically to minimize impact of non-productive times. Condition prediction models considering planned production support the process. This approach is already realized in the field of automotive where maintenance intervals of modern cars are not following fixed time patterns but are rather dependent on drivers driving behaviour and hence the actual vehicle utilization.

Planning simulations and What-If analysis in conjunction with CMMS puts the best plan into action to meet the KPls set forth by the business.

KPI based planning and scheduling simplifies the complex steering process by using a few KPls that have influence on given production targets like "minimize inventory", "maximize throughput". This limited amount of KPIs is orchestrating the full amount of tuning parameters in the background.

Production tracking closes the feedback loop and opens the door for a self-learning planning system that adjusts its internal tuning parameters considering actual vs. originally planned production.

An optimized production is only a valuable asset if the quality of the produced product matches expectations. Complex process environments like steel production always have the potential for quality deviations. Identifying the defects is crucial and the starting point for a deviation management and disposition process, which is ultimately automatic. Such a process is supported by integrating Through Process Optimization with its Through Process Quality Control. Automatic detection of a defect's root cause across all steps of the production process allows the correction of 
product design information- in an ideal case - preventing the defect from happening again. This is possible by integrating mathematical methods like Statistical Process Control (SPC) or big data algorithms. Steering the influencing parameters of product design with the results of those analysis leads to a closed quality loop.

\section{Summary}

The digitalization of steel production will be achieved by integration of sensors, automation and IT systems in several dimensions. A maximum degree of automation and the local optimization of each production facility can be reached by vertical integration. Smart sensors provide information to implement additional automatic functions and enable cyber-physical systems, which play a major role in vertical integration. Condition monitoring and furthermore condition prediction reduce downtime and support predictive maintenance to reduce maintenance costs.

The optimization along the entire production chain - the horizontal integration - is carried out by three groups of systems: Production Management, managing and optimizing the production and interfacing to the ERP system, Through-Process Optimization optimizing the production process and the product quality and finally a Computerized Maintenance Management System supporting all aspects of maintenance. These systems will grow together and interact to further optimize quality, flexibility and productivity.

Along the entire life-cycle of a plant, from engineering to decommissioning, all relevant data about the plant will be made available to operators and maintenance staff in a context-oriented way to support tasks and decisions in the best possible way.

The high degree of interaction and the availability of large amounts of information about the production process combined with digitalized Through-Process Know-how will allow the systems to make autonomous, production related decisions.

The benefit of digitalization for steel producers is better product quality, more flexibility in production and increase productivity.

\section{REFERENCES}

[1] S. Jeschke, C. Brecher, T. Meisen, D. Özdemir, T. Eschert, Industrial Internet of Things, Springer International Publishing, 2017

[2] H. Kagermann, W. Wahlster, J. Helbig. "Umsetzungsempfehlungen für das Zukunftsprojekt Industrie 4.0." Abschlussbericht Arbeitsgruppe Industrie 4.0 (2013).

[3] VDMA, Made in China 2025, VDMA 2016

[4] G. Winter, Industry 4.0 - we are a part of it, stahl+eisen, 2016/3, pp 137. 